Expected number of locally maximal solutions for random Boolean CSPs 


\title{
A Note on the Approximation of Perpetuities
}

\author{
Margarete Knape and Ralph Neiningerf] \\ Department for Mathematics and Computer Science, J.W. Goethe-University Frankfurt, \\ 60054 Frankfurt a. M., Germany \\ received $17^{\text {th }}$ February 2007, revised $19^{\text {th }}$ January 2008 , accepted .
}

We propose and analyze an algorithm to approximate distribution functions and densities of perpetuities. Our algorithm refines an earlier approach based on iterating discretized versions of the fixed point equation that defines the perpetuity. We significantly reduce the complexity of the earlier algorithm. Also one particular perpetuity arising in the analysis of the selection algorithm Quickselect is studied in more detail. Our approach works well for distribution functions. For densities we have weaker error bounds although computer experiments indicate that densities can also be well approximated.

\section{Contents}

$\begin{array}{lll}1 & \text { Introduction } & 124\end{array}$

\begin{tabular}{|lll}
2 & The general approach and organization of the paper & 125
\end{tabular}

\begin{tabular}{|lll}
\hline 3 & Discrete approximation & 127
\end{tabular}

\begin{tabular}{|lll}
4 & Convergence of the discrete approximations & 127
\end{tabular}

\begin{tabular}{|lll}
5 & Algorithm and Complexity & 129
\end{tabular}

\begin{tabular}{|lll}
6 & A simple class of perpetuities & 131
\end{tabular}

7 Example: Number of key exchanges in Quickselect 132

\section{Introduction}

A perpetuity is a random variable $X$ in $\mathbb{R}$ that satisfies the stochastic fixed-point equation

$$
X \stackrel{d}{=} A X+b,
$$

where the symbol $\stackrel{d}{=}$ denotes that left and right hand side in (1) are identically distributed and where $(A, b)$ is a vector of random variables being independent of $X$, whereas dependence between $A$ and $b$ is allowed.

\footnotetext{
${ }^{\dagger}$ Supported by an Emmy Noether Fellowship of the Deutsche Forschungsgemeinschaft. 
The term perpetuity originated in the context of insurance and financial mathematics, where $X$ represents the value of a commitment to make regular payments, $b$ representing the payment and $A$ a discount factor both being subject to random fluctuation; see, e.g. Goldie and Maller (2000) or Embrechts, Klüppelberg, and Mikosch (1997, Section 8.4). However, perpetuities arise in various different contexts: In discrete mathematics, they come up as the limit distributions of certain count statistics of decomposable combinatorial structures such as random permutations or random integers. In these areas, perpetuities often arise via relationships to the GEM and Poisson-Dirichlet distributions; see Arratia, Barbour, and Tavaré (2003) for perpetuities, GEM and Poisson-Dirichlet distribution in the context of combinatorial structures; see Donnelly and Grimmett (1993) for occurrences in probabilistic number theory.

In the probabilistic analysis of algorithms, perpetuities arise as limit distributions of certain cost measures of recursive algorithms such as the selection algorithm Quickselect, see e.g. Hwang and Tsai (2002) or Mahmoud, Modarres, and Smythe (1995).

As perpetuities are given implicitly by their fixed-point characterization (1), properties of their distributions are not directly amenable. Nevertheless various questions about perpetuities have already been settled. Necessary and sufficient conditions on $(A, b)$ for the fixed-point equation (1) to uniquely determine a probability distribution are discussed in Vervaat (1979) and Goldie and Maller (2000). The types of distributions possible for perpetuities have been identified in Alsmeyer, Iksanov, and Rösler (2007). Tail behavior of perpetuities has been studied for certain cases in Goldie and Grübel (1996).

In the present extended abstract, we are interested in the central region of the distributions. The aim is to algorithmically approximate perpetuities, in particular their distribution functions and their Lebesgue densities (if they exist).

For this, we apply and refine an approach proposed in Devroye and Neininger (2002) that was originally designed for random variables $X$ satisfying distributional fixed-point equations of the form

$$
X \stackrel{d}{=} \sum_{r=1}^{K} A_{r} X^{(r)}+b,
$$

where $X^{(1)}, \ldots, X^{(K)},\left(A_{1}, \ldots, A_{K}, b\right)$ are independent with $X^{(r)}$ being identically distributed as $X$ for $r=1, \ldots, K$ and random coefficients $A_{1}, \ldots, A_{K}, b$, and $K \geq 2$.

The case of perpetuities, i. e. $K=1$, structurally differs from the cases $K \geq 2$ : The presence of more than one independent copy of $X$ on the right hand side in (2) often has a smoothing effect so that under mild additional assumptions on $\left(A_{1}, \ldots, A_{K}, b\right)$ the existence of smooth Lebesgue densities of $X$ follows, see Fill and Janson (2000) and Devroye and Neininger (2002). On the other hand, the case $K=1$ often leads to distributions $\mathcal{L}(X)$ that have no smooth Lebesgue density; an example is discussed in Section 7 .

\section{The general approach and organization of the paper}

A random variable $X$ satisfies the distributional identity (1) if and only if its distribution is a fixed-point of the map $T$ on the space $\mathcal{M}$ of probability distributions, given by

$$
T: \mathcal{M} \rightarrow \mathcal{M}, \mu \mapsto \mathcal{L}(A Y+b),
$$

where $Y$ is independent of $(A, b)$, and $\mathcal{L}(Y)=\mu$. Under the conditions $\|A\|_{p}<1$ and $\|b\|_{p}<\infty$ for some $p \geq 1$, which we assume throughout the paper, this map is a contraction on certain complete metric 
subspaces of $\mathcal{M}$. Hence, $\mathcal{L}(X)$ can be obtained as limit of iterations of $T$, starting with some distribution $\mu_{0}$.

However, it is not generally possible to algorithmically compute the iterations of $T$ exactly. We therefore use discrete approximations $\left(A^{(n)}, b^{(n)}\right)$ of $(A, b)$, which become more accurate for increasing $n$, to approximate $T$ by a mapping $\widetilde{T}^{(n)}$, defined by

$$
\widetilde{T}^{(n)}: \mathcal{M} \rightarrow \mathcal{M}, \mu \mapsto \mathcal{L}\left(A^{(n)} Y+b^{(n)}\right),
$$

where again $Y$ is independent of $\left(A^{(n)}, b^{(n)}\right)$ and $\mathcal{L}(Y)=\mu$.

To allow for an efficient computation of the approximation, we impose a further discretization step $\langle\cdot\rangle_{n}$, explained in more detail below, defining

$$
T^{(n)}: \mathcal{M} \rightarrow \mathcal{M}, \mu \mapsto \mathcal{L}\left(\left\langle A^{(n)} Y+b^{(n)}\right\rangle_{n}\right),
$$

where $Y$ is independent of $\left(A^{(n)}, b^{(n)}\right)$ and $\mathcal{L}(Y)=\mu$.

In Section 3, we give conditions for $T^{(n)} \circ T^{(n-1)} \circ \cdots \circ T^{(1)}\left(\mu_{0}\right)$ to converge to the perpetuity given as the solution of (11). To this aim, we derive a rate of convergence in the minimal $L_{p}$ metric $\ell_{p}$, defined on the space $\mathcal{M}_{p}$ of probability measures on $\mathbb{R}$ with finite absolute $p$-th moment by

$$
\ell_{p}(\nu, \mu):=\inf \left\{\|V-W\|_{p}: \mathcal{L}(V)=\nu, \mathcal{L}(W)=\mu\right\}, \quad \text { for } \nu, \mu \in \mathcal{M}_{p}
$$

where $\|\cdot\|_{p}$ denotes the $L_{p}$-norm of random variables. To get an explicit error bound for the distribution function, we then convert this into a rate of convergence in the Kolmogorov metric $\rho$, defined by

$$
\rho(\nu, \mu):=\sup _{x \in \mathbb{R}}\left|F_{\nu}(x)-F_{\mu}(x)\right|,
$$

where $F_{\nu}, F_{\mu}$ denote the distribution functions of $\nu, \mu \in \mathcal{M}_{p}$. This implies explicit rates of convergence for distribution function and density, depending on the corresponding moduli of continuity of the fixedpoint.

For these moduli of continuity we find global bounds for perpetuities with $b \equiv 1$ in Section 6 . For cases with random $b$, these moduli of continuity have to be derived individually. One example, related to the selection algorithm Quickselect, is worked out in detail in Section7

We analyze the complexity of our approach in Section 5 . As a measure for the complexity of the approximation, we use the number of steps needed to obtain an accuracy of order $O(1 / n)$. Although we generally follow the approach in Devroye and Neininger (2002), we can improve the complexity significantly by using different discretizations. For the approximation of the distribution function to an accuracy of $O(1 / n)$ in a typical case, we obtain a complexity of $O\left(n^{1+\varepsilon}\right)$ for any $\varepsilon>0$. In comparison, the algorithm described in Devroye and Neininger (2002), which originally was designed for fixed-point equations of type (2) with $K \geq 2$, would lead to a complexity of $O\left(n^{4+\varepsilon}\right)$, if applied to our cases. For the approximation of the density to an accuracy of order $O(1 / n)$, we obtain a complexity of $O\left(n^{1+1 / \alpha+\varepsilon}\right)$ for any $\varepsilon>0$ in the case of $\alpha$-Hölder continuous densities, cf. Corollary 5.2 .

Proofs are omitted throughout this extended abstract and can be found in Knape (2006). 


\section{Discrete approximation}

Recall that our basic assumption in equation (1) is that $\|A\|_{p}<1$ and $\|b\|_{p}<\infty$ for some $p \geq 1$. To obtain an algorithmically computable approximation of the solution of the fixed-point equation (1), we use an approximation of the sequence defined as follows: We replace $(A, b)$ by a sequence of independent discrete approximations $\left(A^{(n)}, b^{(n)}\right)$, converging to $(A, b)$ in $p$-th mean for $n \rightarrow \infty$. To reduce the complexity, we introduce a further discretization step $\langle\cdot\rangle_{n}$, which reduces the number of values attained by $X_{n}$ :

$$
\widetilde{X}_{n}:=A^{(n)} X_{n-1}+b^{(n)}, \quad X_{n}:=\left\langle\widetilde{X}_{n}\right\rangle_{n} .
$$

In Section 5, explicit discretizations are given, but here we only assume that the discretizations $A^{(n)}, b^{(n)}$ and $\langle\cdot\rangle_{n}$ satisfy

$$
\left\|A^{(n)}-A\right\|_{p} \leq R_{A}(n), \quad\left\|b^{(n)}-b\right\|_{p} \leq R_{b}(n), \quad\left\|\left\langle\widetilde{X}_{n}\right\rangle_{n}-\tilde{X}_{n}\right\|_{p} \leq R_{X}(n),
$$

for some error functions $R_{A}, R_{b}$ and $R_{X}$, which we specify later.

Furthermore, we assume that there exists some $\xi_{p}<1$, such that for all $n \geq 1$,

$$
\left\|A^{(n)}\right\|_{p} \leq \xi_{p}
$$

which in applications is easy to obtain under $\|A\|_{p}<1$ and (6).

\section{Convergence of the discrete approximations}

By arguments similar to those used in Fill and Janson (2002) and Devroye and Neininger (2002) we obtain the following rates for the convergence of the approximations $X_{n}$ towards the fixed-point $X$. We use the shorthand notation $\ell_{p}(X, Y):=\ell_{p}(\mathcal{L}(X), \mathcal{L}(Y))$.

Lemma 4.1 Let $\left(X_{n}\right)$ be defined by (5) and $\xi_{p}$ as in (7). Then

$$
\ell_{p}\left(X_{n}, X\right) \leq \xi_{p}^{n}\|X-\mathbb{E} X\|_{p}+\sum_{i=0}^{n-1} \xi_{p}^{i} R(n-i),
$$

where $R(n):=R_{X}(n)+R_{A}(n)\|X\|_{p}+R_{b}(n)$ for the error functions in (6).

To make these estimates explicit we have to specify functions that bound $R_{A}(n), R_{b}(n)$, and $R_{X}(n)$. We do so in two different ways, one representing a polynomial discretization of the corresponding random variables and one representing an exponential discretization. Better asymptotic results are obtained by the latter one.

Corollary 4.2 Let $\left(X_{n}\right)$ be defined by (5) and $\xi_{p}$ as in (7), and assume

$$
R_{A}(n) \leq C_{A} \frac{1}{n^{r}}, \quad R_{b}(n) \leq C_{b} \frac{1}{n^{r}}, \quad R_{X}(n) \leq C_{X} \frac{1}{n^{r}}
$$


for some $r \geq 1$. Then, we have

$$
\ell_{p}\left(X_{n}, X\right) \leq C_{r} \frac{1}{n^{r}}
$$

where

$$
C_{r}:=\frac{r\|X-\mathbb{E} X\|_{p}}{e^{r} \log \left(1 / \xi_{p}\right)}+\frac{r !\left(C_{X}+C_{b}+C_{A}\|X\|_{p}\right)}{\left(1-\xi_{p}\right)^{r+1}} .
$$

Remark 4.3 In Corollary 4.2 we are merely interested in the order of magnitude of $\ell_{p}\left(X_{n}, X\right)$ without a sharp estimate of the constant $C_{r}$. When evaluating the error in an explicit example, we directly use, $c f$. Lemma 4.1.

$$
\ell_{p}\left(X_{n}, X\right) \leq \xi_{p}^{n}\|X-\mathbb{E} X\|_{p}+\left(C_{X}+C_{A}\|X\|_{p}+C_{b}\right) \sum_{i=0}^{n-1} \frac{\xi_{p}^{i}}{(n-i)^{r}}
$$

to obtain sharper estimates.

Corollary 4.4 Let $\left(X_{n}\right)$ be defined by (5) and $\xi_{p}$ as in (7), and assume

$$
R_{A}(n) \leq C_{A} \frac{1}{\gamma^{n}}, \quad R_{b}(n) \leq C_{b} \frac{1}{\gamma^{n}}, \quad R_{X}(n) \leq C_{X} \frac{1}{\gamma^{n}}
$$

for some $1<\gamma<1 / \xi_{p}$. Then, we have

$$
\ell_{p}\left(X_{n}, X\right) \leq C_{\gamma} \frac{1}{\gamma^{n}}
$$

where

$$
C_{\gamma}:=\|X-\mathbb{E} X\|_{p}+\frac{\left(C_{X}+C_{b}+C_{A}\|X\|_{p}\right)}{1-\xi_{p} \gamma} .
$$

Lemma 4.5 Let $X_{n}$ and $C_{r}$ be as in Corollary 4.2 and $X$ have a bounded density $f_{X}$. Then, the distance in the Kolmogorov metric can be bounded by

$$
\rho\left(X_{n}, X\right) \leq\left(C_{r}(p+1)^{1 / p}\left\|f_{X}\right\|_{\infty}\right)^{p /(p+1)} n^{-r p /(p+1)} .
$$

Similarly, for $X_{n}$ and $C_{\gamma}$ as in Corollary 4.4 we have

$$
\rho\left(X_{n}, X\right) \leq\left(C_{\gamma}(p+1)^{1 / p}\left\|f_{X}\right\|_{\infty}\right)^{p /(p+1)} \gamma^{p n /(p+1)} .
$$

Remark 4.6 In some cases, we can give a similar bound, although the density of $X$ is not bounded or no explicit bound is known. Instead, it is sufficient to have a polynomial bound for the modulus of continuity of the distribution function $F_{X}$ of $X$, cf. Knape (2006). 
To approximate the density of the fixed-point, we define

$$
f_{n}(x)=\frac{F_{n}\left(x+\delta_{n}\right)-F_{n}\left(x-\delta_{n}\right)}{2 \delta_{n}},
$$

where $F_{n}$ is the distribution function of $X_{n}$. For this approximation we can give a rate of convergence, depending on the modulus of continuity of the density of the fixed-point, which is defined by

$$
\Delta_{f_{X}}(\delta):=\sup _{\substack{u, v \in \mathbb{R} \\|u-v| \leq \delta}}\left|f_{X}(u)-f_{X}(v)\right|
$$

Lemma 4.7 Let $X$ have a density $f_{X}$ with modulus of continuity $\Delta_{f_{X}}$ and let $\left(X_{n}\right)$ be defined by (5). Then, for $f_{n}$ defined by 12 , and all $\delta_{n}>0$,

$$
\left\|f_{n}-f_{X}\right\|_{\infty} \leq \frac{1}{\delta_{n}} \rho\left(X_{n}, X\right)+\Delta_{f_{X}}\left(\delta_{n}\right)
$$

Corollary 4.8 Let $X$ have a bounded density $f_{X}$, which is Hölder continuous with exponent $\alpha \in(0,1]$. For polynomial discretization, i.e. $X_{n}$ and $C_{r}$ as in Corollary 4.2 and $f_{n}$ defined by [12] with

$$
\delta_{n}:=L n^{-r p /((\alpha+1)(p+1))}
$$

with an $L>0$, we have

$$
\left\|f_{n}-f_{X}\right\|_{\infty} \leq\left(\left(C_{r}(p+1)^{1 / p}\left\|f_{X}\right\|_{\infty}\right)^{p /(p+1)} / L+c L^{\alpha}\right) n^{-\alpha r p /((\alpha+1)(p+1))}
$$

For exponential discretization, i.e. $X_{n}$ and $C_{\gamma}$ as in Corollary 4.4 and $f_{n}$ defined by (12) with

$$
\delta_{n}:=L \gamma^{-p n /((\alpha+1)(p+1))}
$$

with an $L>0$, we obtain

$$
\left\|f_{n}-f_{X}\right\|_{\infty} \leq\left(\left(C_{\gamma}(p+1)^{1 / p}\left\|f_{X}\right\|_{\infty}\right)^{p /(p+1)} / L+c L^{\alpha}\right) \gamma^{\alpha p n /((\alpha+1)(p+1))} .
$$

Remark 4.9 If $X$ is bounded and bounds for the density $f_{X}$ and its modulus of continuity are known explicitly, the last result is strong enough to allow, in principle, perfect simulation using von Neumann's rejection method; see Devroye (2001) for the case of infinitely divisible perpetuities with approximation of densities by Fourier inversion, Devroye, Fill, and Neininger (2000) for the case of the Quicksort limit distribution and Devroye and Neininger (2002) for more general fixed-point equations of type (2).

\section{Algorithm and Complexity}

In this section, we will give an algorithm for an approximation satisfying the assumptions in the last section for many important cases. We assume that the distributions of $A$ and $b$ are given by Skorohod representations, i.e. by measurable functions $\varphi, \psi:[0,1] \rightarrow \mathbb{R}$, such that

$$
A=\varphi(U) \quad \text { and } \quad b=\psi(U),
$$


where $U$ is uniformly distributed on $[0,1]$. Furthermore, we assume that $\|\varphi\|_{\infty} \leq 1$ and that both functions are Lipschitz continuous and can be evaluated in constant time. Now we define the discretization $\langle\cdot\rangle_{n}$ by

$$
\langle Y\rangle_{n}:=\lfloor s(n) Y\rfloor / s(n),
$$

where $s(n)$ can be either polynomial, i. e. $s(n)=n^{r}$ or exponential, $s(n)=\gamma^{n}$. Defining

$$
\begin{aligned}
A^{(n)} & :=\varphi\left(\langle U\rangle_{n}\right) \quad \text { and } \\
b^{(n)} & :=\psi\left(\langle U\rangle_{n}\right),
\end{aligned}
$$

the conditions on $\varphi$ and $\psi$ ensure that Corollary 4.2 and 4.4 can be applied.

We keep the distribution of $X_{n}$ in an array $\mathcal{A}_{n}$, where $\mathcal{A}_{n}[k]:=\mathbb{P}\left[X_{n}=k / s(n)\right]$ for $k \in \mathbb{Z}$. Note however, that as $A$ and $b$ are bounded, $\mathcal{A}_{n}[k]=0$ at least for $|k|>Q_{n}$, where $Q_{n}$ can be computed recursively as $Q_{n}=\|A\|_{\infty} Q_{n-1}+\|b\|_{\infty}$ and $Q_{0}=\left\|X_{0}\right\|_{\infty}=\mathbb{E}[X]$. The core of the implementation is the following update procedure:

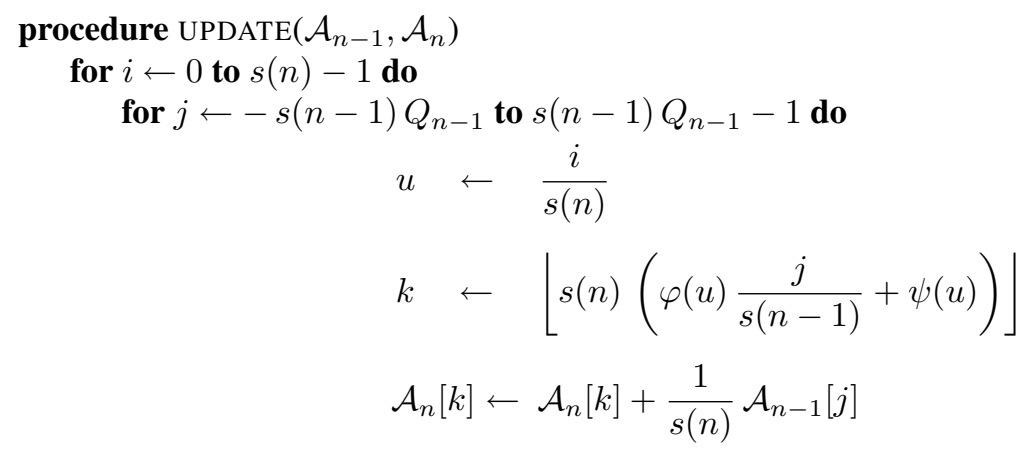

end for

end for

end procedure

The complete code for polynomial discretization for the example in Section 7, implemented in C++, can be found in Knape (2006).

To approximate the density as in 12 with $\delta_{n}=d / s(n)$ for some $d \in \mathbb{N}$, we compute a new array $\mathcal{D}_{n}$ by setting

$$
\mathcal{D}_{n}[k]=\frac{s(n)}{2 d} \sum_{j=k-d+1}^{k+d} \mathcal{A}_{n}[j] .
$$

To measure the complexity of our algorithm, we estimate the number of steps needed to approximate the distribution function and the density up to an accuracy of $O(1 / n)$. For the case that $X$ has a bounded density $f_{X}$ which is Hölder continuous, we give asymptotic bounds for polynomial as well as for exponential discretization. We assume the general condition (13).

Lemma 5.1 Assume that $X$ has a bounded density $f_{X}$, which is Hölder continuous with exponent $\alpha \in$ $(0,1]$. Using polynomial discretization with exponent $r, c f$. Corollary 4.2 we can approximate the distribution function of $X$ to an accuracy of $O(1 / n)$ in time $O\left(n^{(2+2 / r)(p+1) / p}\right)$ and the density $f_{X}$ to the same accuracy in time $O\left(n^{2(1+1 / \alpha)(r+1)(p+1) /(r p)}\right)$. 
Using exponential discretization with parameter $\gamma$ as in Corollary 4.4. approximation to the same accuracy takes time $O\left(n^{(p+1) / p} \log n\right)$ for the distribution function of $X$. The density can be approximated to the same accuracy in time $O\left(n^{(1+1 / \alpha)(p+1) / p} \log n\right)$.

Corollary 5.2 Assume that $X$ has a bounded density $f_{X}$, which is Hölder continuous with exponent $\alpha \in(0,1]$. Then, using exponential discretization as in Corollary 4.4 approximation to an accuracy of $O(1 / n)$ takes time $O\left(n^{1+\varepsilon}\right)$ for the distribution function and time $O\left(n^{1+1 / \alpha+\varepsilon}\right)$ for the density of $X$ for all $\varepsilon>0$.

\section{A simple class of perpetuities}

In order to make the bounds of Section 4 explicit in applications, we need to bound the absolute value and modulus of continuity of the density of the fixed-point. For a simple class of fixed-point equations, we give universal bounds in this section. For more complicated cases, bounds have to be derived individually, which we work out for one example in Section 7 .

For fixed-point equations of the form

$$
X \stackrel{d}{=} A X+1 \quad \text { with } A \geq 0
$$

where $A$ and $X$ are independent, we can bound the density and modulus of continuity of $X$ using the corresponding values of $A$.

Lemma 6.1 Let $X$ satisfy fixed-point equation (15) and A have a density $f_{A}$. Then $X$ has a density $f_{X}$ satisfying

$$
f_{X}(u)=\int_{1}^{\infty} \frac{1}{x} f_{A}\left(\frac{u-1}{x}\right) f_{X}(x) d x, \quad \text { for } u \geq 1,
$$

and $f_{X}(u)=0$ otherwise.

Corollary 6.2 Let A have a bounded density $f_{A}$. Then $X$ has a density $f_{X}$ satisfying

$$
\left\|f_{X}\right\|_{\infty} \leq\left\|f_{A}\right\|_{\infty}
$$

Corollary 6.3 Let $A$ have a density $f_{A}$, and $\Delta_{f_{A}}$ be its modulus of continuity. Then the modulus of continuity $\Delta_{f_{X}}$ of $f_{X}$ satisfies

$$
\Delta_{f_{X}}(\delta) \leq \Delta_{f_{A}}(\delta) \quad \delta>0
$$

This result is only useful if the density of $A$ is continuous, but we can extend it to many practical examples, where $f_{A}$ has jumps at points in a countable set $\mathcal{I}_{A}$. We use the jump function of $f_{A}$, defined by

$$
J_{f_{A}}(s)=f_{A}(s)-\lim _{x \uparrow s} f_{A}(x), \quad s>0
$$

and a modification of $f_{A}$ where we remove all jumps,

$$
\bar{f}_{A}:=f_{A}-\sum_{s \in \mathcal{I}_{A} \backslash\{0\}} J_{f_{A}}(s) \mathbb{1}_{[s, \infty)} .
$$

Since $X \geq 1$, we now denote by $\Delta_{f_{X}}$ the modulus of continuity of the restriction of $f_{X}$ to $(1, \infty)$. 
Lemma 6.4 Let A have a bounded càdlàg density $f_{A}$. Then, for all $\delta>0$,

$$
\Delta_{f_{X}}(\delta) \leq \Delta_{\bar{f}_{A}}(\delta)+\left\|f_{X}\right\|_{\infty} \sum_{s \in \mathcal{I}_{A} \backslash\{0\}} \frac{\left|J_{f_{A}}(s)\right| \delta}{s} .
$$

\section{Example: Number of key exchanges in Quickselect}

In this section, we apply our algorithm to the fixed-point equation

$$
X \stackrel{d}{=} U X+U(1-U),
$$

where $U$ and $X$ are independent and $U$ is uniformly distributed on $[0,1]$. This equation appears in the analysis of the selection algorithm Quickselect. The asymptotic distribution of the number of key exchanges executed by Quickselect when acting on a random equiprobable permutation of length $n$ and selecting an element of rank $k=o(n)$ can be characterized by the above fixed-point equation, see Hwang and Tsai (2002).

We use our algorithm to get a discrete approximation of the fixed point. The plot of a histogram, generated with $n=80, r=3$, can be found in Figure 1 .

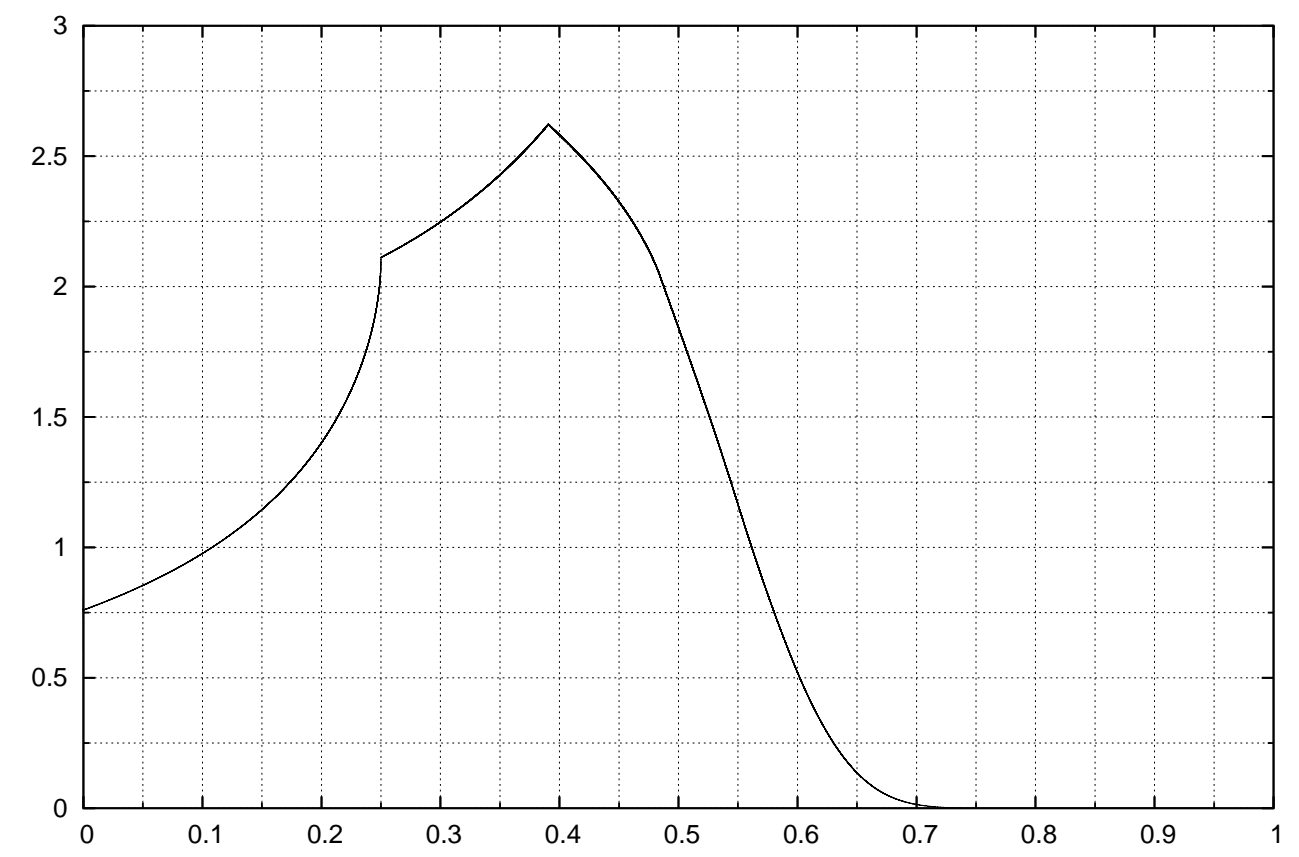

Fig. 1: Histogram of an approximation of $X$ with $X \stackrel{d}{=} U X+U(1-U)$.

In the following, we specify how the bounds in Section 4 can be made explicit for this example. 
Lemma 7.1 Let $X$ be a solution of 17). Then, we have $0 \leq X \leq 1$ almost surely, and the moments are recursively given by $\mathbb{E}\left[X^{0}\right]=1$ and

$$
\mathbb{E}\left[X^{k}\right]=(k+1) !(k-1) ! \sum_{j=0}^{k-1} \frac{\mathbb{E}\left[X^{j}\right]}{j !(2 k-j+1) !}, \quad k \geq 1,
$$

in particular, $\mathbb{E}[X]=1 / 3$.

Lemma 7.2 Let $X$ be a solution of (17). Then, for all $\kappa \in \mathbb{N}$ and $\varepsilon>0$,

$$
\mathbb{P}[X \geq 1-\varepsilon] \leq 2^{\left(\kappa^{2}-\kappa\right) / 4} \varepsilon^{\kappa / 2} .
$$

Lemma 7.3 Let $X$ be a solution of (17). Then $X$ has a Lebesgue density $f$ satisfying $f(t)=0$ for $t<0$ or $t>1$ and

$$
f(t)=2 \int_{p_{t}}^{t} g(x, t) f(x) d x+\int_{t}^{1} g(x, t) f(x) d x \quad \text { for } t \in[0,1],
$$

where

$$
p_{t}:=2 \sqrt{t}-1, \quad g(x, t):=\frac{1}{\sqrt{(1+x)^{2}-4 t}} .
$$

Remark 7.4 For $t=0$, equation 18 implies

$$
f(0)=\mathbb{E}\left[\frac{1}{1+X}\right]=0.759947956 \ldots
$$

In order to use Lemma 4.5 to bound the deviation of our approximation, we need an explicit bound for the density of $X$. We derive a rather rough bound here and will see later, that we can use the resulting bound for our approximation to improve it.

Lemma 7.5 Let $f$ be the density of $X$ as in Lemma 7.3. Then

$$
\|f\|_{\infty} \leq 18
$$

Lemma 7.6 Let $f$ be the density of $X$ as in Lemma 7.3. Then $f$ is Hölder continuous on [0, 1] with Hölder exponent $\frac{1}{2}$ :

$$
|f(t)-f(s)| \leq 9\|f\|_{\infty} \sqrt{t-s}, \quad \text { for } 0 \leq s<t \leq 1 .
$$

Remark 7.7 The latter Lemma cannot be substantially improved, as in $t=1 / 4$, the density $f(t)$ is not Hölder continuous with Hölder exponent $1 / 2+\varepsilon$ for any $\varepsilon>0$.

\section{Explicit error bounds}

We can now combine the bounds for the density and its modulus of continuity with Lemma 4.5 and Lemma 4.7 to bound the deviation of an approximation from the solution of the fixed-point equation.

To approximate the density $f$ we set

$$
f_{n}(x):= \begin{cases}f(0) & \text { for } 0 \leq x \leq \delta_{n}, \\ \frac{F_{n}\left(x+\delta_{n}\right)-F_{n}\left(x-\delta_{n}\right)}{2 \delta_{n}} & \text { for } \delta_{n}<x \leq 1 \\ 0 & \text { otherwise, }\end{cases}
$$


where $F_{n}$ is the distribution function of $X_{n}$ and $f(0)$ is given in Remark 7.4

With $r=3$, cf. Corollary 4.2 , and $n=80$ we obtain:

Corollary 7.8 We have $\rho\left(X_{80}, X\right) \leq 1.162 \cdot 10^{-4}$, and $\left\|f_{80}-f\right\|_{\infty} \leq 0.931$. Furthermore, we can improve the bound of Lemma 7.5 and get $\|f\|_{\infty} \leq 3.561$.

Remark 7.9 Using the realistic (but yet unproven) bound of $\|f\|_{\infty} \leq 2.7$ we would obtain $\rho\left(X_{80}, X\right) \leq$ $8.9809 \cdot 10^{-5}(p=13)$ and $\left\|f_{80}-f\right\|_{\infty} \leq 0.7101$. Hence, our approach works well for the distribution function. However, we cannot show strong error bounds for the approximation of densities with our arguments.

In Table 1, the resulting error bounds for several possible discretizations with similar running time can be found.

\begin{tabular}{|c|c||c|c||c|}
\hline Discret. & $N$ & $\rho\left(X_{N}, X\right)$ & opt. $p$ & $s(N)$ \\
\hline \hline$n$ & 22000 & 0.00178 & 14 & 22000 \\
\hline$n^{2}$ & 430 & 0.00025 & 16 & 184900 \\
\hline$n^{3}$ & 80 & 0.00012 & 13 & 512000 \\
\hline$n^{4}$ & 30 & 0.00050 & 3 & 810000 \\
\hline \hline $1.5^{n}$ & 35 & 0.00070 & 3 & 1456110 \\
\hline $1.7^{n}$ & 27 & 0.00187 & 2 & 1667712 \\
\hline
\end{tabular}

Tab. 1: Bounds for $\rho\left(X_{n}, X\right)$ for comparable total running times. The discretizations are according to Corollaries 4.2 and 4.4 and $s(N)$ denotes the number of atoms of the discrete approximation.

In Knape (2006, Section 4), the algorithm is applied to several other fixed-point equations for which the solutions are more or less explicitly known and the actual error can be evaluated. It appears that the error bounds in Section 4 are rather loose and that the approximation is much better than indicated by our bounds.

\section{References}

G. Alsmeyer, A. Iksanov, and U. Rösler. On distributional properties of perpetuities. Preprint, 2007.

R. Arratia, A. D. Barbour, and S. Tavaré. Logarithmic combinatorial structures: a probabilistic approach. EMS Monographs in Mathematics. European Mathematical Society (EMS), Zürich, 2003.

L. Devroye. Simulating perpetuities. Methodol.Comp. Appl. Probab., 3:97-115, 2001.

L. Devroye and R. Neininger. Density approximation and exact simulation of random variables that are solutions of fixed-point equations. Adv. Appl. Prob., 34:441-468, 2002.

L. Devroye, J. A. Fill, and R. Neininger. Perfect simulation from the Quicksort limit distribution. Elect. Comm. in Probab., 5:95-99, 2000.

P. Donnelly and G. Grimmett. On the asymptotic distribution of large prime factors. J. London Math. Soc., 47:395-404, 1993. 
P. Embrechts, C. Klüppelberg, and T. Mikosch. Modelling extremal events. For insurance and finance. Number 33 in Applications of Mathematics (New York), 33. Springer-Verlag, Berlin, 1997.

J. A. Fill and S. Janson. Smoothness and decay properties of the limiting Quicksort density function. In Mathematics and Computer Science: Algorithms, Trees, Combinatorics and Probabilities (Versailles, 2000), pages 53-64. Birkhäuser Verlag, 2000.

J. A. Fill and S. Janson. Quicksort asymptotics. J. Algorithms, 44:4-28, 2002.

C. Goldie and R. Grübel. Perpetuities with thin tails. Adv. Appl. Probab., 28:463-480, 1996.

C. Goldie and R. Maller. Stability of perpetuities. Ann. Probab., 28:1195-1218, 2000.

H.-K. Hwang and T.-H. Tsai. Quickselect and the Dickman function. Comb. Probab. Comput., 11:353$371,2002$.

M. Knape. Approximating perpetuities. Diploma thesis, J.W. Goethe-Universität Frankfurt a.M., 2006. URL http://publikationen.ub.uni-frankfurt.de/volltexte/2007/3859/

H. Mahmoud, R. Modarres, and R. Smythe. Analysis of QUICKSELECT: an algorithm for order statistics. RAIRO Inform. Théor. Appl., 29:255-276, 1995.

W. Vervaat. On a stochastic difference equation and a representation of non-negative infinitely divisible random variables. Adv. Appl. Prob., 11:750-783, 1979. 
